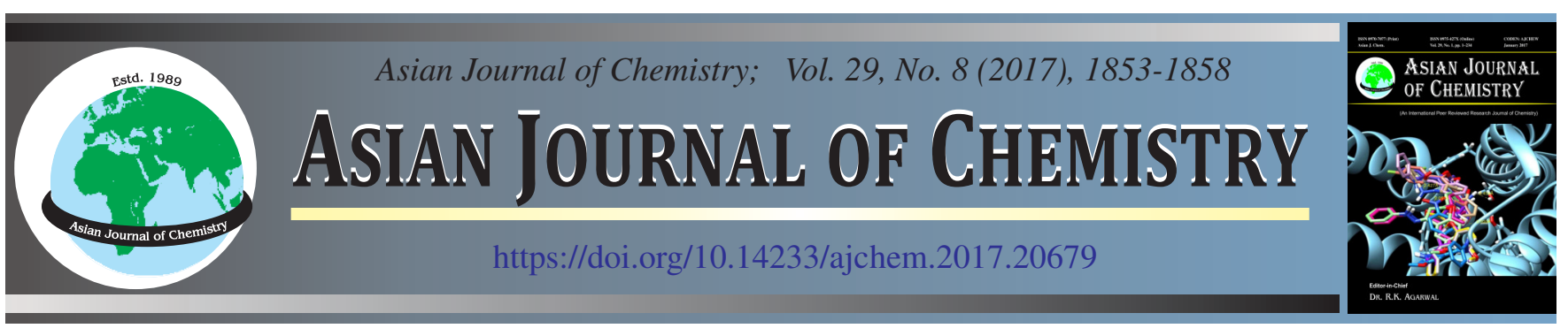

\title{
An Orthogonal Approach for Method development, Validation and Trace Level Quantification of 2,5-Diamino-4,6-dichloro Pyrimidine Impurity in Abacavir Sulfate by LC-MS/MS and GC-MS
}

\author{
Nagaraju Rajana ${ }^{1,2, *}$, P. Madhavan ${ }^{1}$, H. Rama Mohan ${ }^{1}$, J. Moses Babu ${ }^{3}$ and K. Basavaiah ${ }^{2}$
}

${ }^{1}$ Technology Development Center, Custom Pharmaceutical Services, Dr. Reddy's Laboratories Ltd., Miyapur, Hyderabad-500 049 , India ${ }^{2}$ Department of Inorganic \& Analytical Chemistry, Andhra University, Visakhapatnam-530 003, India

${ }^{3}$ Integrated Product Development Organization, Dr. Reddy's Laboratories Ltd, Innovation Plaza, Bachupally, Hyderabad-500 072, India

*Corresponding author: E-mail: nagarajurajana@drreddys.com; nagarajrajana@gmail.com

The trace level quantification of 2,5-diamino-4,6-dichloro pyrimidine (DADCP) present in abacavir sulfate by LC-MS/MS and GC-MS has been proposed. LC-MS/MS method was established for $2.5 \mathrm{ppm}$ with respect to test concentration $(10 \mathrm{mg} / \mathrm{mL}), \mathrm{LOQ}$ and LOD were established as 1.3 and $0.4 \mathrm{ppm}$, respectively. Linearity, method precision, accuracy at LOQ, $100 \%, 150 \%$, solution, mobile phase stability and robustness parameters were studied against the ICH Q2(R1) guideline. Similarly, the GC-MS method was established for 2.5 ppm of 2,5-diamino-4,6-dichloro pyrimidine with respect to test concentration (50 mg/mL), LOQ and LOD were established as 1.1 and $0.4 \mathrm{ppm}$, respectively. Linearity, method precision, accuracy at LOQ, $100 \%, 150 \%$, solution stability and robustness parameters were studied against the ICH Q2 (R1). No analytical method was found in trace level quantification of 2,5-diamino-4,6-dichloro pyrimidine in abacavir sulfate. The orthogonal method of LC-MS/MS and GC-MS was well robust and can be used for determination of trace level of 2,5-diamino-4,6-dichloro pyrimidine in different pharmaceutical compounds.

Keywords: Quantification, 2,5-Diamino-4,6-dichloro pyrimidine, Abacavir sulfate, LC-MS/MS, GC-MS.

\section{INTRODUCTION}

Abacavir $(\mathrm{ABC})$ is an antiretroviral medication used to prevent and treat HIV/AIDS [1]. It is a synthetic carbocyclic nucleoside and is the enantiomer with $1 \mathrm{~S}, 4 \mathrm{R}$ absolute configuration on the cyclopentene ring. Vivo, abacavir sulfate dissociates to its free base abacavir [2]. The administration of abacavir at $600 \mathrm{mg}$ once daily and $300 \mathrm{mg}$ twice daily in human immunodeficiency virus-infected subjects [3]. And abacavir prevents to halt the inroads of the human immunodeficiency virus (HIV). Without treatment, HIV gradually undermines the body's immune system, encouraging other infections to take hold until the body succumbs to full-blown acquired immune deficiency syndrome (AIDS). Initially, abacavir is phosphorylated to its corresponding monophosphate as an intracellular reaction. Cytosolic enzymes convert abacavir monophosphate to carbovir monophosphate (CBV-MP), which is finally phosphorylated to the biologically active moiety, carbovir triphosphate (CBV-TP). CBV-TP inhibits HIV reverse transcriptase by competing with the endogenous substrate DGTP and by chain termination subsequent to incorporation into the growing polynucleotide strand [4]. DADCP impurity is the structural alert for the potential impurity in the abacavir sulfate and it needs to be control less than the $2.5 \mathrm{ppm}$ in the final drug substance and drug product. But so far no method was provided for low quantification of DADCP potential impurity.

Many analytical methods were developed to determine the drug substance and drug product of abacavir sulfate and its process and degradation impurities like the UPLC method for determination of abacavir sulfate its related substances and degradation products [5]. HPLC method for assay of abacavir sulfate, determination of abacavir sulfate, its related substances and degradation products are available in literature. Stereo selectivity of abacavir sulfate [6], determination of abacavir in plasma, simultaneous determination with other antiretroviral products, human serum and biological matrices [7-14]. Electrochemical method for determination of abacavir sulfate [15], mass spectrometric method for determination of abacavir sulphate, its related substance and degradation products and its characterization are known [16-18].

Objective of this present work is to quantify the trace level of 2,5-diamino-4,6-dichloro pyrimidine (DADCP) in abacavir sulfate by using LC-MS/MS and GC-MS in the way 
of orthogonal method. 2,5-Diamino-4,6-dichloro pyrimidine is the impurity $[19,20,22]$ which is part of synthetic process in abacavir sulphate, no method was available for trace level quantification of 2,5-diamino-4,6-dichloro pyrimidine (DADCP) in abacavir sulphate. LC-MS/MS method and GC-MS methods are specific to all process and degradation impurities.

\section{EXPERIMENTAL}

Abacavir sulfate (99.9\% w/w) and 2,5-diamino-4,6dichloro pyrimidine (DADCP) $(98.0 \%)$ were provided by the process research department of Custom Pharmaceutical Services of Dr. Reddy's laboratories limited, Hyderabad, India. HPLC-grade of Acetonitrile Rankem (Mumbai, India) used for the studies. Millipore Milli Q plus (Bangalore, India) purification system was used to prepare high pure water.

LC-MS/MS: Method development of impurity in abacavir sulfate was done by using Agilent 1100 series LC system with UV detector and connected Agilent MS 6410 QqQ instrument equipped with ESI source and triple quadrupole analyzer (City, CA USA), software used to data analysis is Mass Hunter. MS/MS was done by using nitrogen gas collision gas. By doing DADCP fragmentation, MRM pair was selected.

GC-MS: Methods development of impurity present in abacavir sulfate was done by using Agilent 7890A Gas chromatograph equipped with $5975 \mathrm{C}$ mass selective detector. EI source with single quadrupole used to do quantification and selected ion monitoring used for the selection of ions and quantification of impurity. The software used to evaluate the data is Mass Hunter quantification software with 21 CFR part 11 compliance.

Standard solution preparation for LC-MS/MS and GCMS: In LC-MS/MS, the standard solution were prepared by weighing $10 \mathrm{mg}$ of DADCP and transferred into a $100 \mathrm{~mL}$ volumetric flask, dissolved in $10 \mathrm{~mL}$ of acetonitrile: water $(50: 50 \% \mathrm{v} / \mathrm{v})$ and made up to the mark with diluent for stock solution-1. Transferred $0.5 \mathrm{~mL}$ of stock solution-1 into a $50 \mathrm{~mL}$ volumetric flask and made upto the mark with diluent. Transferred $0.33,0.48,0.62,0.95$ and $1.25 \mathrm{~mL}$ of stock solution-2 into five different $25 \mathrm{~mL}$ volumetric flask to prepare 1.3, 1.9, 2.5, 3.8 and 5. 0 ppm of standard solutions with respect to analyte concentration $(10 \mathrm{mg} / \mathrm{mL})$. Standard solutions were using for calibration curve method to determine the content of the DADCP in the abacavir sulfate. In GC-MS, the standard solution were prepared by weighing $10 \mathrm{mg}$ of DADCP and transferred in a $100 \mathrm{~mL}$ volumetric flask, dissolved by $10 \mathrm{~mL}$ of acetonitrile and made up to the mark with diluent for stock solution-1. Transferred $1 \mathrm{~mL}$ of stock solution- 1 into a 100 $\mathrm{mL}$ volumetric flask and made upto the mark with diluent. Transferred 1.5, 2.2, 3.1, 4.51 and $6.0 \mathrm{~mL}$ of stock solution-2 into five different $25 \mathrm{~mL}$ volumetric flasks to prepare 1.3, 1.9, 2.5, 3.8 and $5.0 \mathrm{ppm}$ of standard solutions with respect to analyte concentration $(50 \mathrm{mg} / \mathrm{mL})$. Standard solutions were using for calibration curve method to determine the content of the DADCP in the abacavir sulfate by LC-MS/MS and GCMS.

The following formula used for calculation of DADCP content.

Content of DADCP in ppm $=(\mathrm{Y}-\mathrm{C} / \mathrm{m}) *$ (weight taken/ actual weight), where, $\mathrm{Y}=$ area of test sample, $\mathrm{C}=$ intercept, $\mathrm{m}=$ slope, $\mathrm{Wt}$. = weight of test sample taken in $\mathrm{mg}$ or By using the mass hunter quantitative analysis software and plot the linearity curve by taking concentration on $\mathrm{X}$-axis and area on Y-axis, software will be reported the content of the DADCP present in abacavir sulfate sample.

Sample preparation: Sample preparation was simple and easy procedure for LC-MS/MS and GC-MS. In LC-MS/MS. The sample have been prepared by taking $100 \mathrm{mg}$ of abacavir sulfate sample weighed and transferred into $10 \mathrm{~mL}$ volumetric flask and dissolved in $3 \mathrm{~mL}$ of 50:50 mixture of acetonitrile and water and made up to the mark with diluent and mix well. This solution was directly used for analysis. Similarly in GCMS the sample has been prepared by taking $500 \mathrm{mg}$ of abacavir sulfate, sample was weighed and transferred into $10 \mathrm{~mL}$ volumetric flask and dissolved in $3 \mathrm{~mL}$ of acetonitrile. The sample was not completely soluble, mixed $10 \mathrm{~min}$ by using cyclomixer and filtered with $0.45 \mu$ filter and injected into GC-MS system.

Preparation of spiked solution: Spiked solution of DADCP in abacavir sulfate were prepared by taking standard solution at LOQ, $2.5 \mathrm{ppm}$ and $3.8 \mathrm{ppm}$ into separate $10 \mathrm{~mL}$ volumetric flasks and added $100 \mathrm{mg}$ of abacavir sulfate solution in method of LC-MS/MS and $500 \mathrm{mg}$ abacavir sulfate in method of GCMS, dissolved by standard solutions and made upto the mark with standard solutions.

\section{RESULTS AND DISCUSSION}

MS/MS conditions: Initial MS/MS method development was started with direct mass in the LC-MS with single quadrupole. The mass of DADCP was observed in positive mode of ionization in ESI as $179[\mathrm{M}+\mathrm{H}]^{+}$with dichloro pattern after subtracting background of the system in scan mode i.e., 100 a.m.u. to 1600 a.m.u [Fig. 1(A)]. The base peak mass is molecular ion of DADCP. $179[\mathrm{M}+\mathrm{H}]^{+}$is the precursor ion for the MS/MS study. The collision energy at $10 \mathrm{eV}$ product ion of $179[\mathrm{M}+\mathrm{H}]^{+}$was $143.10 \mathrm{~m} / \mathrm{z}$ stable fragment [Fig. 1(B)]. But still $179[\mathrm{M}+\mathrm{H}]^{+}$was not vanished at $35 \mathrm{eV}$ also and at $50 \mathrm{eV}$ product ion was the 89 which was stable ion and the 179 $[\mathrm{M}+\mathrm{H}]^{+}$was vanished [Fig. $\left.1(\mathrm{C})\right]$. Similarly the abacavir sulfate was injected into Q1, the $287[\mathrm{M}+\mathrm{H}]^{+}$mass observed [Fig. 1(D)]. No such fragments found as like DADCP in abacavir sulfate. Fragmentor for all this study was 135 , temperature was $350^{\circ} \mathrm{C}$, gas flow was $10 \mathrm{~L} / \mathrm{min}$ and the capillary voltage for the study was 4000 volts, EMV played critical role in low level detection of DADCP at 300 volts of EMV, it was detected.

LC conditions: Initial LC method development was started with Zorbax C18, $150 \mathrm{~mm} .4 .6 \mathrm{~mm}$ and $3.5 \mu \mathrm{m}$ size columns the separation of DADCP and abacavir were closely eluted. For better separation and peak sharpness YMC pack pro C18 $150 \mathrm{~mm} \times 4.6 \mathrm{~mm}$ and $3.0 \mu \mathrm{m}$ column was used, in order to get the short method for quantification. The less particle size column was taken and both DADCP and abacavir are polar should be separated. The ratio of organic and aqueous also having effect on the separation of DADCP and abacavir sulfate. The mobile phase chosen for this study was $0.1 \%$ formic acid in water and acetonitrile, initial study was studied with different ratio of water and acetonitrile, at $50 \%$ of $0.1 \%$ formic acid in water and acetonitrile with isocratic elution the separation of 

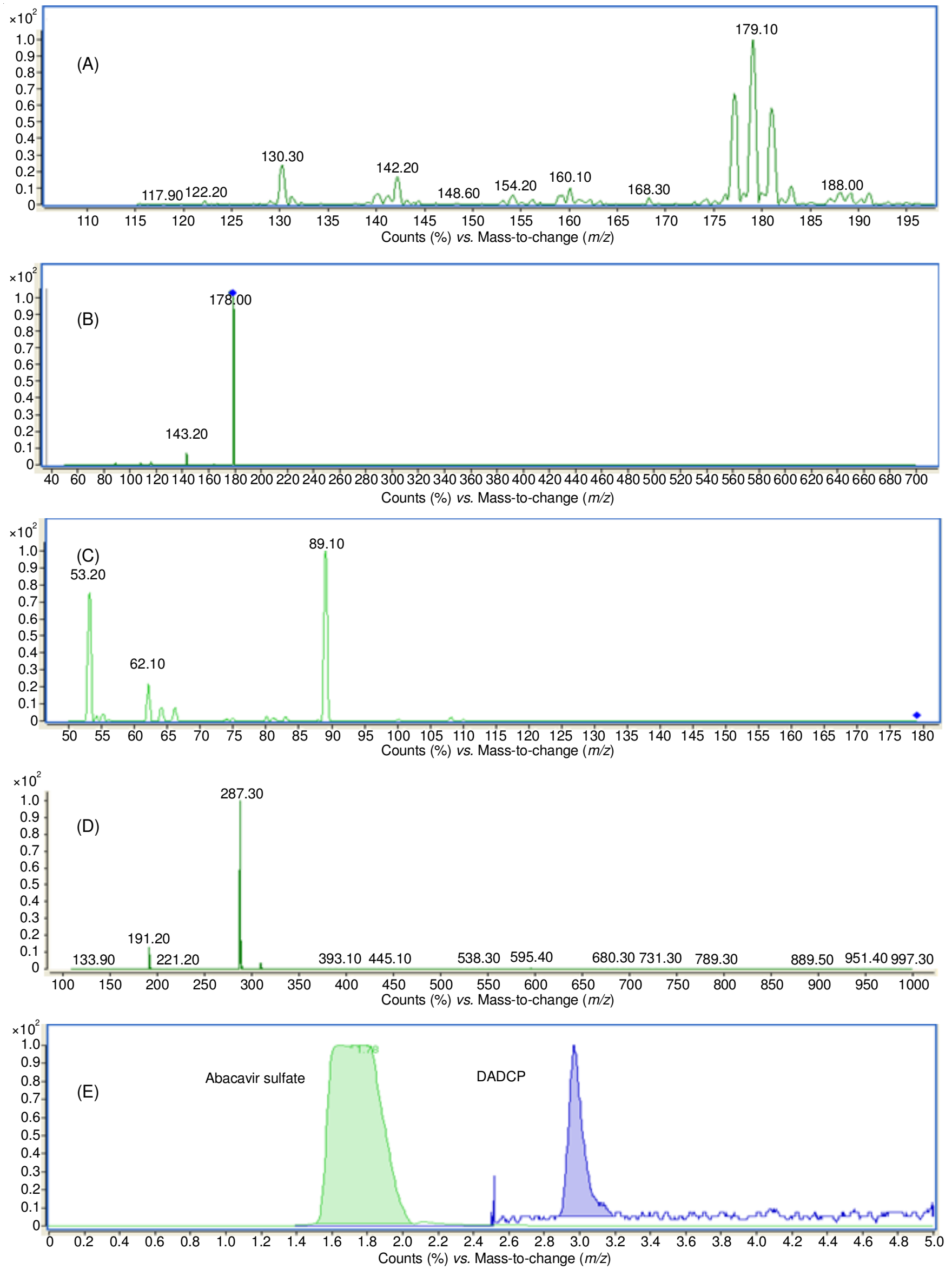

Fig. 1. (A)ESI mass spectrum of DADCP and MS/MS spectra of $m / z, 179[\mathrm{M}+\mathrm{H}]^{+}$ion at (B) MS/MS spectra of $m / z 179[\mathrm{M}+\mathrm{H}]^{+}$ion at $10 \mathrm{eV}$ (C) $50 \mathrm{eV}$ for DADCP (D) ESI mass spectrum of abacavir sulfate (E) VWD chromatogram of abacavir sulfate and TIC of DADCP 
DADCP and abacavir were well separated [Fig. 1(E)]. The temperature of column oven was studied but no variation it the retention time and response, by change of flow, the peak of DADCP coming close to the abacavir sulfate hence the flow was optimized to $0.7 \mathrm{~min}$. Finally ambient temperature was chosen for the final quantifications.

GC-MS conditions: GC-MS method development trails have been started with solubility of abacavir sulfate drug substance, solubility with DMSO,DMA were more compare to low boilers e.g. methanol, acetonitrile, dichloro methane, etc. With respect to $50 \mathrm{mg} / \mathrm{mL}$, the solubility of abacavir sulfate was not there in low boilers and but at lower analyte solubility, the required level response is not sufficient to show the LOQ and LOD as well as the recovery. $50 \mathrm{mg} / \mathrm{mL}$ concentration was chosen to start the development of the DADCP in abacavir sulfate. The column stationary phase have started with nonpolar to mid polar such as DB-1,HP-5 and DB-1701, DADCP is mid polar hence the retention time and the peak shape was good in DB-1701 column, split ratio, injection volume, oven temperature program, injector temperature and auxiliary temperature were optimized. The mass conditions were initiated with scan mode; the ionized, abundant mass was selected for selected ion monitoring [Fig. 2(A) \& (B)].

\section{Optimization of LC-MS/MS and GC-MS method}

LC-MS/MS: LC and mass conditions were optimized, the liquid chromatography optimized conditions were run time with $5.0 \mathrm{~min}$, mobile phase containing $0.1 \%$ formic acid in $50: 50 \mathrm{v} / \mathrm{v} \%$ acetonitrile: water, flow was $0.8 \mathrm{~mL} / \mathrm{min}$, load of injection was $10 \mu \mathrm{L}$, column was YMC Pack pro C18 $150 \mathrm{~mm}$ $\times 4.6 \mathrm{~mm}, 3.0 \mu \mathrm{m}$, column temperature was $25^{\circ} \mathrm{C}$, concentration of analyte for response of impurity was $10 \mathrm{mg} / \mathrm{mL}$. MS/MS spectrometry condition were optimized and MS/MS optimized condition were capillary voltage as 4000 volts, source temperature was 300 temperature, gas flow $10 \mathrm{~L} / \mathrm{h}$ and fragmentor 135 [Fig. 2(C)].

GC-MS: Gas chromatography and mass spectrometric conditions were optimized to reach the $2.5 \mathrm{ppm}$ as specification with respect to API concentration $50 \mathrm{mg} / \mathrm{mL}$. The simple gas chromatographic conditions were optimized with DB-1701 column as with $30 \mathrm{~m} \times 250 \mu \mathrm{m} \times 0.25 \mu \mathrm{m}$ and injection volume as $2 \mu \mathrm{L}$, Helium flow was $1.2 \mathrm{~mL} / \mathrm{min}$, split ratio was $5: 1$, injector temperature was $250{ }^{\circ} \mathrm{C}$, auxiliary temperature was $265^{\circ} \mathrm{C}$, oven temperature program was $150{ }^{\circ} \mathrm{C}$ for 0 min hold then $5^{\circ} \mathrm{C} / \mathrm{min}$ ramp to $250{ }^{\circ} \mathrm{C}$ for $2 \mathrm{~min}$ hold, the total run time was $22 \mathrm{~min}$ according to oven program. The optimized EI-MS conditions were selected, mass for the selected ion monitoring was 142.9, $177.9,179.9$, the mass selected mass abundant mass gave enough response to quantify the impurity of its limit $(2.5 \mathrm{ppm})$, the solvent delay was $6.8 \mathrm{~min}$, gain factor was 1.0, EMV was 1541, timed events off was $9.0 \mathrm{~min}$, resolution was low mode, the tune file was auto tune and the Column\#2 used to connect mass and gas chromatography with $1 \mathrm{~m} \times 180 \mu \mathrm{m} \times 0 \mu \mathrm{m}$ because no stationary for this column, this is working like as interface between gas chromatography and mass spectrometry [Fig. 2(D)].

\section{Validation}

Specificity: Specificity is the ability to assess unequivocally the analyte in the presence of components which may be expected to be present. Typically these might include impurities, degradants, matrix as per ICHQ2 (R1) [21], etc. Specificity in mass spectrometric methods, the impurity is main focused component and the impurity should be specified from the other impurities and active pharmaceutical ingredients. The specificity study was done by spiking the DADCP in abacavir sulfate and injected in LC-MS as well as in GC-MS; no other mass number was interfered with the DADCP. In the LC-MS method of abacavir sulfate peak was eluted before the DADCP hence the abacavir sulfate eluted run time kept in the waste by mass parameter, but in GC-MS the peak was not eluted because the boiling point of abacavir sulfate was more than $250{ }^{\circ} \mathrm{C}$, it is not enter the gas chromatography by injector. Hence, both methods were specifying with active pharmaceutical ingredients and other impurities.

Linearity: The linearity of an analytical procedure is its ability (within a given range) to obtain test results which are directly proportional to the concentration (amount) of analyte in the sample. Mass detectors are near linear detector hence the range of solutions were kept from 1.3-5.0 $\mu \mathrm{g} / \mathrm{mL}$ for both LC-MS/MS and GC-MS methods and the correlation coefficient were found to be 0.997 and 0.998 , respectively (Table-1).

\begin{tabular}{lcc}
\multicolumn{3}{c}{ TABLE-1 } \\
LC-MS/MS AND GC-MS VALIDATION \\
\multicolumn{1}{c}{ RESULTS OF DADCP IN ABACAVIR SULFATE } \\
\hline \multicolumn{1}{c}{ Parameter } & LC-MS/MS & GC-MS \\
\hline LOQ in ppm w.r.t. analyte concentration & 1.3 & 1.1 \\
LOD in ppm w.r.t. analyte concentration & 0.4 & 0.4 \\
Slope (m) & 112.4 & 3448 \\
Intercept (c) & 38.1 & 731 \\
Correlation coefficient(r) & 0.997 & 0.998 \\
Method precision (\% RSD) & 2.8 & 6.3 \\
LOQ precision (\% RSD) & 4.3 & 4.4 \\
Intermediate precision (\% RSD) & 1.3 & 3.3 \\
Accuracy at 1.3 ppm (\% Recovery) & 100.1 & 109.5 \\
Accuracy at 2.5 ppm (\% Recovery) & 95.2 & 107.2 \\
Accuracy at 3.8 ppm (\% Recovery) & 101.4 & 112.7 \\
Solution stability and mobile phase diffe- & 0.4 & 0.1 \\
rence with respect to initial (\% variation) & & \\
\hline w.r.t. = with respect to test sample & &
\end{tabular}

Precision: The precision of an analytical procedure expresses the closeness of agreement (degree of scatter) between a series of measurements obtained from multiple sampling of the same homogeneous sample under the prescribed conditions. Method precision and intermediate precision were established and method precision results were meeting the ICH Q2 R1 guidelines and \% RSD of the DADCP in abacavir sulfate was found to be $2.8 \%$ at $2.5 \mathrm{ppm}$ by LC-MS/MS and $6.3 \%$ at 2.5 ppm by GC-MS. The intermediate precision was done by different day, different column, different analyst and different lots of chemicals. The \% RSD were found to be 1.3 and 3.0 by LC-MS and GC-MS, respectively (Table-1).

Accuracy: The accuracy of an analytical procedure expresses the closeness of agreement between the value which is accepted either as a conventional true value or an accepted reference value and the value found. Accuracy of DADCP impurity in abacavir sulfate at $2.5 \mathrm{ppm}$ and $3.8 \mathrm{ppm}$ were studied. The \% recovery at $2.5 \mathrm{ppm}$ was found to be 95.2 and 

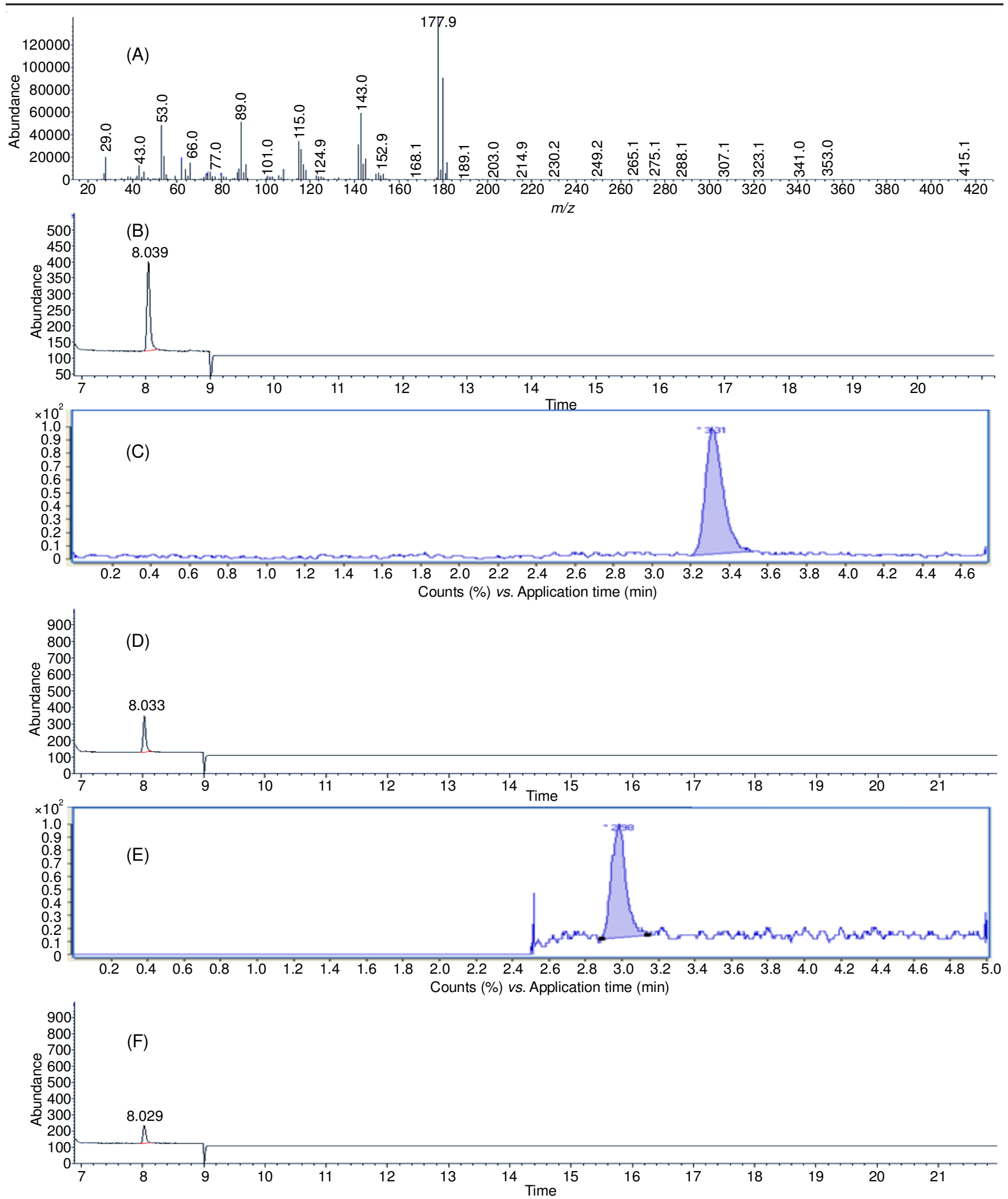

Fig. 2. (A) Mass spectrum of DADCP by GC-MS (B) GC-MS Total ion chromatogram (C) 2.5 ppm Total ion chromatogram of LC-MS/MS (D) $2.5 \mathrm{ppm}$ Total ion chromatogram of GC-MS (E) LOQ Total ion chromatogram of LC-MS/MS (F) LOQ Total ion chromatogram of GC-MS

$107.2 \%$ in LC-MS/MS and GC-MS, respectively. The \% recovery at 3.8 ppm was 101.4 and $112.7 \%$ in LC-MS/MS and GC-MS, respectively (Table-1).

Limit of quantitation, limit of detection, precision and accuracy at limit of quantitation: The quantitation limit of an individual analytical procedure is the lowest amount of analyte in a sample which can be quantitatively determined with suitable precision and accuracy. The quantitation limit is a parameter of quantitative assays for low levels of compounds in sample matrices. The LOQ and LOD were establishment 
by using STYEX method. The linearity solution were kept for the establishment for LOQ and LOD from $1.3 \mathrm{ppm}$ to $5.0 \mathrm{ppm}$.

The LOQ values were found to be $1.3 \mathrm{ppm}$ and $1.1 \mathrm{ppm}$ in LC-MS/MS and GC-MS, respectively (Table-1). LOQ precision was studied and the precision limits were within the limits as per ICH Q2 R1. The \%RSD of LOQ precision of DADCP in abacavir sulfate by LC-MS and GC-MS were 4.3 and $4.4 \%$, respectively, The LOD values were three times less than the LOQ values 0.4 ppm was LOD in the LC-MS/ MS and GC-MS [Fig. 2(E) \& (F)].

Solution stability and mobile phase stability: Solution stability and mobile phase stability were studied for the DADCP in presence of the abacavir sulfate. The DADCP as $2.5 \mathrm{ppm}$ was spiked in abacavir sulfate and injected into LC-MS/MS with interval of $12 \mathrm{~h}$ and obtained $100 \%$ recovery of content of DADCP in the abacavir sulfate and with same mobile phase $100 \%$ recovery of content of DADCP in the abacavir sulfate, similarly $100 \%$ recovery of DADCP content in the abacavir sulfate by GC-MS in acetonitrile diluent.

Robustness: The evaluation of robustness should be considered during the development phase and depends on the type of procedure under study. It should show the reliability of an analysis with respect to deliberate variations in method parameters. In LC-MS/MS. the flow, temperature, mobile phase variation have been done and linear robust parameters applied to the method, its robust with different temperature and linear robust with flow, variation in mobile phase concentration and MRM pair. In GC-MS, the method was robust by change in flow and column with different equivalents.

\section{Conclusion}

An orthogonal approach for LC-MS/MS and GC-MS quantitative method was developed for quantitation of DADCP impurity in abacavir sulfate at $2.5 \mathrm{ppm}$ level. Both liquids and gas chromatography with mass detection were used to detect the trace level quantification and method was specific towards the process impurities and volatile solvents, so far no orthogonal method was used for the determination of the DADCP impurity in abacavir sulfate active pharmaceutical ingredient and other pharmaceutical compounds. Availability of any one of these two instruments in GMP lab, the DADCP can be analyzed. All validation parameters such as system suitability, linearity, LOQ, LOD, method precision, intermediate precision, accuracy, solution stability, mobile phase stability and robustness met the predefined acceptance criteria as per ICH Q2 (R1).

\section{ACKNOWLEDGEMENTS}

The authors thank Dr. Reddy's Laboratories Ltd., Hyderabad, India, for providing the facilities to carry out this study. Cooperation from colleagues of Analytical Research \&
Development and process research and development of Dr. Reddy's Laboratories Ltd. is thankfully acknowledged.

\section{REFERENCES}

1. Abacavir Sulfate, The American Society of Health-System Pharmacists, Retrieved 31 July 2015.

2. http://www.drugbank.ca/drugs/DB01048.

3. G. Moyle, M. Boffito, C. Fletcher, C. Higgs, P.E. Hay, I.H. Song, Y. Lou, G.J. Yuen, S.S. Min and E.M. Guerini, Antimicrob. Agents Chemother, 53, 1532 (2009);

https://doi.org/10.1128/AAC.01000-08.

4. J.R. Huff, Bioorg. Med. Chem., 7, 2667 (1999); https://doi.org/10.1016/S0968-0896(99)00242-4.

5. P. Vukkum, G.R. Deshpande, J.M. Babu, R. Muralikrishna and P. Jagu, Sci. Pharm., 80, 903 (2012); https://doi.org/10.3797/scipharm.1206-11.

6. Pavani Jagu, P. Vukkum1, B.M. Rao and N.S. Rao, Int. J. Pharm. Life Sci., 4, 3043 (2013).

7. M.S. Goizman, T.D. Balayants, G.B. Tikhomirova and N.N. Sal'nikova, Pharm. Chem. J., 46, 187 (2012); https://doi.org/10.1007/s11094-012-0757-5.

8. C.P.W.G.M. Verweij-van Wissen, R.E. Aarnoutse and D.M. Burger, J. Chromatogr. A, 816, 121 (2005);

https://doi.org/10.1016/j.jchromb.2004.11.01.

9. A.I. Veldkamp, R.W. Sparidans, R.M.W. Hoetelmans and J.H. Beijnen, J. Chromatogr. B Biomed. Sci. Appl., 736, 123 (1999); https://doi.org/10.1016/S0378-4347(99)00457-0.

10. U. Seshachalam, B. Haribabu and K.B. Chandrasekhar, J. Sep. Sci., 30, 28 (2007); https://doi.org/10.1002/jssc. 200600209.

11. Y. Ozkan, A. Savaser and S.A. Ozkan, J. Liq. Chromatogr. Rel. Technol., 28, 423 (2005); https://doi.org/10.1081/JLC-200044523.

12. S.M. Ferrer, P. Modamio, C.F. Lastra and E.L. Mariño, Biomed. Chromatogr., 18, 862 (2004); https://doi.org/10.1002/bmc.406.

13. C.P.W.G.M. Verweij-van Wissen, R.E. Aarnoutse and D.M. Burger, J. Chromatogr. B Analyt. Technol. Biomed. Life Sci., 816, 121 (2005); https://doi.org/10.1016/j.jchromb.2004.11.019.

14. T. Tol, N. Kadam, N. Raotole, A. Desai and G. Samanta, J. Chromatogr. A, 1432, 26 (2016); https://doi.org/10.1016/j.chroma.2015.12.080.

15. B. Dogan, B. Uslu, S.A. Ozkan and P. Zuman, Anal. Chem., 80, 209 (2008); https://doi.org/10.1021/ac0713151.

16. S.A.B. Shah, R. Mullin, G. Jones, I. Shah, J. Barker, A. Petroczi and D.P. Naughton, J. Pharm. Biomed. Anal., 74, 308 (2013); https://doi.org/10.1016/j.jpba.2012.10.023.

17. R.N. Rao, R.M. Vali, B. Ramachandra and S.S. Raju, J. Pharm. Biomed. Anal., 54, 279 (2011);

https://doi.org/10.1016/j.jpba.2010.08.021.

18. J.M. Rice and G.O. Dudek, J. Am. Chem. Soc., 89, 2719 (1967); https://doi.org/10.1021/ja00987a039.

19. ICH Q1A(R2), Stability Testing of New Drug Substances and Products, (2000).

20. ICH Q3A(R2), Current Step 4 Version, Impurities in New Drug Substances, October (2006).

21. ICH Q2(R1), Validation of Analytical Procedures: Text and Methodology, November (2005).

22. M7(R1) ICH Consensus Guideline, Released for Consultation on 9 June (2015). 